EPJ Web of Conferences 73, 01005 (2014)

DOI: $10.1051 /$ epjconf/20147301005

(C) Owned by the authors, published by EDP Sciences, 2014

\title{
The light meson spectroscopy program
}

\author{
Elton S. Smith \\ Thomas Jefferson National Accelerator Facility, Newport News, VA 23606, USA
}

\begin{abstract}
Recent discoveries of a number of unexpected new charmomium-like meson states at the BaBar and Belle B-factories have demonstrated how little is still known about meson spectroscopy. In this talk we will review recent highlights of the light quark spectroscopy from collider and fixed target experiments.
\end{abstract}

\section{Landscape}

Within the quark model, mesons are described as hadrons with the simplest configuration of a quark and anti-quark pair $(q \bar{q})$. This model has been surprisingly successful in explaining the main features of the spectrum despite the fact that mesons are bound states of the complex theory of QCD, which allows for intricate configurations of gluons, quarks and anti-quarks. The simple features of a $q \bar{q}$ system are exhibited more strongly for heavy quark systems, e.g. charm and bottom mesons. However, even there recent discoveries are shinning light on a rich spectrum, unexpected even a few years ago.

Much of the experimental interest in the light meson spectrum is focused on configurations outside the naive quark model, i.e. glueballs (valence gluons only), multi-quark systems and hybrids (valence quarks and gluons). We begin by exploring the mass region around $2 \mathrm{GeV}$ using $\mathrm{J} / \psi$ decays (Sect. 2). The spectrum of $0^{++}$mesons is shown in Fig. 1. There has been a long interesting history to understand the nature of these states, as described in the recent review in Ref. [1]. The grouping of mesons below $1 \mathrm{GeV}$ does not fit easily into the quark model and has for many years been considered a likely set of multi-quark configurations (see for example Ref. [2]). The grouping of states between 1.5 and $1.7 \mathrm{GeV}$ consists mostly of quark-model states, but one too many states together with studies of the decay modes have lead investigators to posit that there is also a glueball that is mixed with the $q \bar{q}$ states. For a review see Ref. [3]. Detailed studies are lacking above this mass region and it is now being studied to search for other glueball configurations.

We then turn our attention to fixed target experiments with pion (Sect. 3) and photon (Sect. 4) beams to study the production of hybrid states. The gluonic fields that confine quarks have been shy to manifest their presence in static properties of hadrons, though their degrees of freedom are present in the QCD Lagrangian. However, a subset of hybrids have an unmistakable experimental signature: a combination of $J^{P C}$ quantum numbers that cannot be created from a quark-antiquark pair alone. Such states are called exotic hybrid mesons, and their quantum numbers provide a useful experimental handle to isolate

\footnotetext{
ae-mail: elton@jlab.org
}

This is an Open Access article distributed under the terms of the Creative Commons Attribution License 4.0, which permits unrestricted use, distribution, and reproduction in any medium, provided the original work is properly cited. 


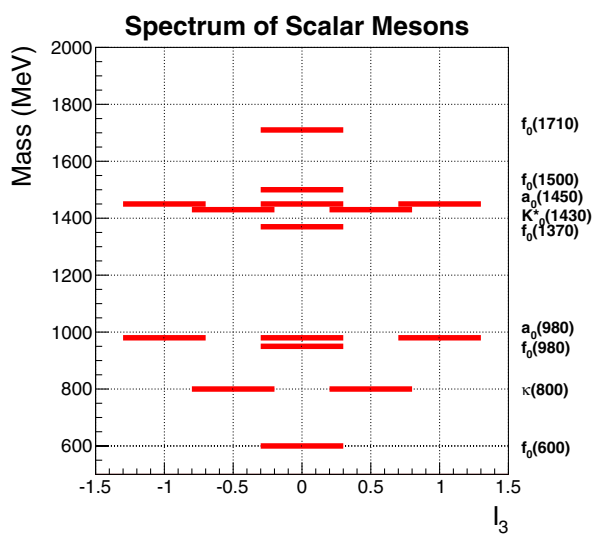

Figure 1. Mass vs. third component of isospin, $I_{3}$, for the low-mass $0^{++}$mesons. The controversial $\kappa(800)$ is included, although it is omitted from the summary table by the Particle Data Group.

special degrees of freedom. Their investigation has therefore become one of the main goals for many programs worldwide. A recent review of the status of these states can be found in Ref. [4].

\section{BESIII (J/\% decays)}

Radiative decays of $\mathrm{J} / \psi$ mesons have historically been considered a good place to search for glueballs because the $c \bar{c}$ pair must decay via annihilation so the intermediate state must have gluons in it. BESIII has been operating at the Beijing Electron-Positron Collider (BEPCII) since 2008 at center-of-mass energies between 2 and $4.4 \mathrm{GeV}$. It has collected 1.2 billion $\mathrm{J} / \psi$ and 0.5 billion $\psi^{\prime}$ to date, but their completed analyses are based on a sample of 200 million J/ $\psi$. The BESIII detector consists of a heliumgas-based drift chamber, a plastic scintillator time-of-flight system and a CsI ( $\mathrm{Tl}$ ) electromagnetic calorimeter, all enclosed in a superconducting solenoidal magnet that provides a $1 \mathrm{~T}$ magnetic field [5]. The solid angle for charged particle and photon acceptance is $93 \%$ of $4 \pi$. A recent review of light meson spectroscopy with BESIII can be found in Ref. [6].

One of the first results of the new BESIII detector is the confirmation [7] of the threshold enhancement of $p \bar{p}$ events in the radiative decay of $J / \psi \rightarrow \gamma X(p \bar{p})$, originally observed by BESII [8]. A spin-parity analysis of the structure has been performed [9] to test various explanations. The analysis includes a sub-threshold $X(p \bar{p})$ resonance, the $f_{2}(1910), f_{0}(2100)$ and $0^{++}$phase space. Fixing the parameters of the $f_{2}$ and $f_{0}$ states, they find the mass of the $X(p \bar{p})$ to be $\mathrm{M}=1832 \pm 19 \mathrm{MeV}$, the width $\Gamma=13 \pm 39 \mathrm{MeV}$, and the branching ratio $B R(J / \psi \rightarrow \gamma X) B R(X \rightarrow p \bar{p}))=9 \pm 1.1 \times 10^{-5}$. The spin-parity analysis strongly favors the $0^{-+}$assignment.

Further understanding of the structure at about $1830 \mathrm{MeV}$ was investigated using $J / \psi \rightarrow \gamma \eta^{\prime} \pi^{+} \pi^{-}$ [10]. This channel is motivated because $\eta^{\prime} \pi^{+} \pi^{-}$is one of the strongest decay modes of the $\eta_{c}$, which may decay like a glueball. Two decay modes of the $\eta^{\prime}$ are considered: $\eta^{\prime} \rightarrow \gamma \rho$ and $\eta^{\prime} \rightarrow \pi^{+} \pi^{-} \eta$. The $\eta^{\prime} \pi^{+} \pi^{-}$ mass distribution is shown in Fig. 2. Prominent in the spectrum are three new structures in addition to the $f_{1}(1510)$ peak at low mass. The fitted mass for the peak in the region of interest is $\mathrm{M}=1837 \pm 3 \mathrm{MeV}$, $\Gamma=190 \pm 9 \mathrm{MeV}$. For these events, an angular distribution of the $\gamma$ in the $J / \psi$ center of mass is consistent with $1+\cos ^{2} \theta$ expected for a pseudoscalar. The higher mass states are intriguing because early pure glue lattice calculations predicted a $0^{-+}$glueball at about $2.6 \mathrm{GeV}$ [11]. However, more complete lattice calculations of the isoscalar sector [12] do not find evidence for glueballs in this mass region. Nevertheless, experimental studies to determine the nature of states in this mass region are of keen interest. 


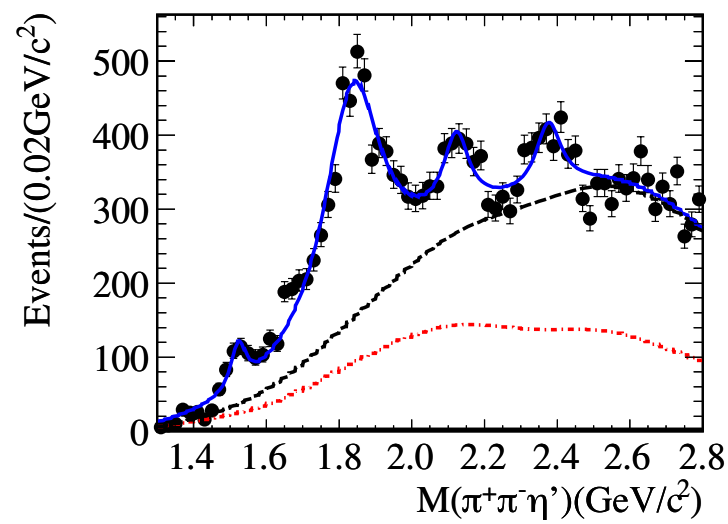

Figure 2. Mass distribution of $X \rightarrow \eta^{\prime} \pi^{+} \pi^{-}$from the radiative decay $J / \psi \rightarrow \gamma X$ from BESIII, Ref. [10]. The dashdotted line is the contribution from misidentification of the signal channel and the dashed line is the contributions of the total background and nonresonant $\eta^{\prime} \pi^{+} \pi^{-}$process.

This mass region has also been studied by looking at the system $X \rightarrow \eta \pi^{+} \pi^{-}$recoiling against an $\omega$ instead of a photon in the decay $J / \psi \rightarrow \omega X$ [13]. The comparison between hadronic decays of the $J / \psi$ with an $\omega$ and its radiative decays could provide important information regarding the possible glueball identification of the $\mathrm{X}(1835)$. The mass spectrum of $\eta \pi^{+} \pi^{-}$shows three prominent peaks. The lower mass peaks are identified as the $f_{1}(1285)$ and $\eta(1405)$ based on their fitted mass, width and specific decay mode of $X \rightarrow a_{0}(980) \pi$, with the subsequent decay of $a_{0} \rightarrow \eta \pi$. The third peak has not yet been identified but has a fitted mass of $\mathrm{M}=1877 \pm 6 \mathrm{MeV}$ and $\Gamma=57 \pm 12 \mathrm{MeV}$. Unfortunately no angular information is available, so the nature of this new state is quite uncertain including whether it can be identified with the $\mathrm{X}(1835)$ observed in $J / \psi$ radiative decays. We await the completion of the analysis of all the data already acquired by the BESIII collaboration to shed light on these structures.

\section{COMPASS $\left(\pi^{-1}\right.$ beam)}

COMPASS is a fixed-target experiment at the Super Proton Synchrotron (SPS) at CERN using $190 \mathrm{GeV} / \mathrm{c}$ muon and hadron beams to study the spin structure of the nucleon and a broad range of topics in QCD including hadron spectroscopy. The spectrometer is equipped with hadronic and electromagnetic calorimeters and charged particle identification is achieved using a RICH detector, which can separate kaons from pions up to $50 \mathrm{GeV} / \mathrm{c}$. The detector has excellent coverage of charged and neutral particles in the center-of-mass of the excited states, selecting reactions with momentum transfers in the range of $0.1<t^{\prime}<1 \mathrm{GeV}^{2} / c^{2}$.

Of particular interest to us is their spectroscopy program, using negative pion beams incident on a variety of targets. A pilot run in 2004 using a $\mathrm{Pb}$ target yielded a first look at the meson spectrum and provided confirmation of the disputed $\pi_{1}(1600)$ exotic state decaying to $\pi^{+} \pi^{-} \pi^{-}$[14]. This analysis was based on approximately 420k events. Subsequent runs in 2008/09 have increased the sample by a factor of 50, allowing increased statistical studies as well as comparison of decays to $\pi^{-} \pi^{0} \pi^{0}$. Data were acquired on a liquid $\mathrm{H}_{2}$ target.

The data are analyzed using partial waves in the isobar model, which decomposes the production and decay of a resonance $X^{-} \rightarrow \pi^{+} \pi^{-} \pi^{-}$and $X^{-} \rightarrow \pi^{-} \pi^{0} \pi^{0}$ into a chain of successive two-body decays. The $\mathrm{X}^{-}$with quantum numbers $J^{P C}$ and spin projection $M^{\epsilon}$ is assumed to decay into a dipion resonance, called the isobar, and a pion, called the bachelor. The isobar has relative orbital angular momentum $L$ with respect to the bachelor pion. Each partial wave is thus specified in the reflectivity 

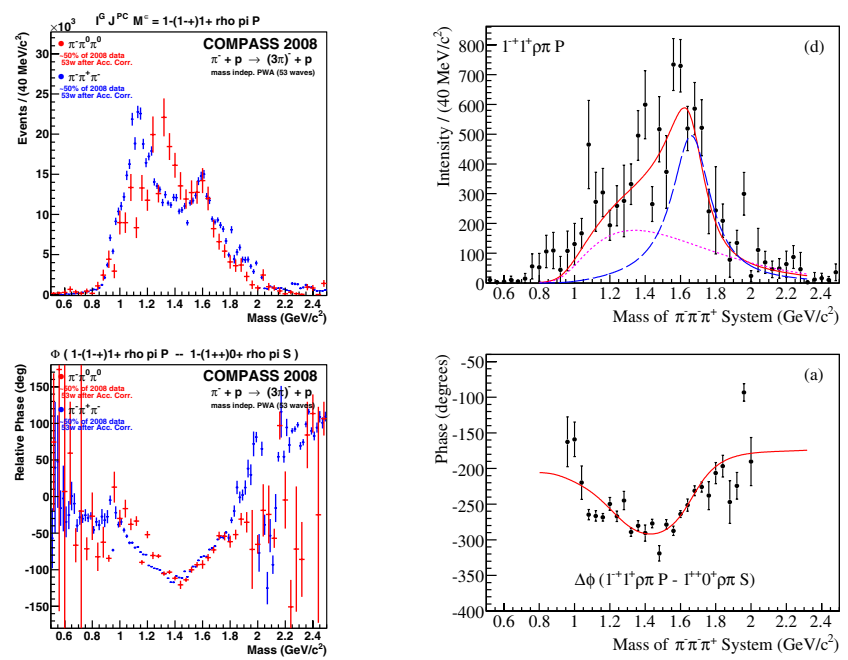

Figure 3. Mass-independent PWA result for the exotic $1^{-+}$wave in $(3 \pi)^{-}$final states for the 2008 data (left). The fitted intensity of $\left(1^{-+}\right) 1^{+} \rho \pi P$ wave is shown for the neutral mode data in comparison to the charged one (top, left), the relative phase difference with respect to the $\left(1^{++}\right) 0^{+} \rho \pi S$ wave for both decay modes is shown as well (bottom, left). The published result on three charged pion final states from the 2004 pilot run data [14] is shown for comparison (right). The figure is reproduced from Ref. [16].

basis using the notation of $J^{P C} M^{\epsilon}[$ isobar $\pi] L$, where $\epsilon= \pm 1$ is the reflectivity. For a description of the partial wave analysis method (PWA) see Ref. [15] and references therein. The analysis is conducted as a two step process; the first step is performed in fixed bins of $3 \pi$ mass using 53 partial waves, 11 more waves than used in the analysis of the 2004 data. The two decay modes are analyzed separately, show good agreement with each other and match the known masses and widths of established resonances in this mass region including the $a_{2}(1320), a_{1}(1260), \pi_{2}(1670)$ and the $a_{4}(2040)$. Consistency is found between the two decay modes when isospin factors are included, and the relative phase motion between these prominent waves is consistent with their resonant behavior.

Figure 3 shows the amplitude of the exotic $1^{-+}$wave and its phase motion relative to the dominant $1^{++} a_{1}(1260)$ for the $2008 / 09$ and 2004 data sets. The amplitudes of the exotic signal show some discrepancy below $1.4 \mathrm{GeV}$ between the two decay modes, but become more consistent in the region identified by other experiments. Moreover, the phase motion of the two decay modes show more consistency and are compatible with resonant behavior for the exotic wave. We note that the intensity in the exotic wave is only a few percent of the total intensity, whereas relative phases are independent of the size in intensity and are therefore a more powerful tool for establishing the structure and the nature in this mass range.

\section{CLAS ( $\gamma$ beam)}

The CLAS detector [17] is based on a toroidal magnetic field and operated in Hall B at Jefferson Lab using electron and photon beams. Six detector segments are instrumented between the magnet coils and function as independent magnetic spectrometers. Broad coverage is provided for particle detection, limited only by the space occupied by the coils. Charged particles are detected with drift chambers and identified as pions using a time-of-flight system. Calorimetry in the forward direction is used for triggering and reconstruction of photons. Early data from CLAS saw no evidence for an exotic $\pi_{1}(1600)$ resonance in photoproduction [18]. We describe a new experiment, which tagged photons in the range 

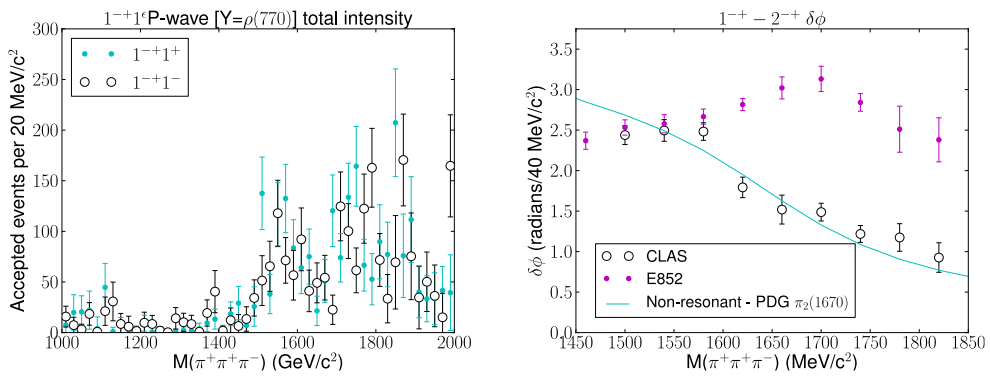

Figure 4. On the left, the intensities of the $1^{-+}, \mathrm{M}=1$ waves are plotted, showing no clear evidence for resonant structure, as is true for the $1^{-+}, \mathrm{M}=0$ wave (not shown). On the right is an overlay of the $1^{-+}-2^{-+}$phase motion for both the CLAS results and those reported by E852 in Ref. [20]. One can see a clear turning-over of the E852 phase, indicative of interference between two Breit-Wigner forms. The CLAS has a steady decrease, indicative of a resonanting $\pi_{2}$ being subtracted from a nonresonant $1^{-+}$, as shown by the curve. The figure is reproduced from Ref. [21].

of 3.6-5.5 GeV and recorded a total of 26.2 billion triggers, corresponding to an integrated luminosity of $68 \mathrm{pb}^{-1}$ [19].

Theoretical models have posited that photoproduction would be a fruitful place to search for hybrid mesons (see discussion and citations in Ref. [4]), which motivated the search for exotics in the reaction $\gamma p \rightarrow n \pi^{+} \pi^{+} \pi^{-}$. The experiment searched for the $1^{-+}$partial wave decaying by $X^{+} \rightarrow \pi^{+} \pi^{+} \pi^{-}$ $[21,22]$. At these energies, the overlap of meson and baryon resonances is substantial and kinematic cuts were made to increase the purity of the meson resonances for the analysis. After making selection cuts on photon energy $\left(E_{\gamma}>4.4 \mathrm{GeV}\right)$, momentum transfer to the $3 \pi$ system $\left(t^{\prime}<0.1 \mathrm{GeV}^{2}\right)$, and laboratory angle of the slow pion $\left(\theta\left[\pi_{\text {slow }}^{+}\right]<25^{\circ}\right)$, the event sample for analysis was $500 \mathrm{k}$ events. These cuts enhanced meson production relative to baryon production and provided an event sample that could be used in a PWA decomposition of the meson spectrum. The masses and widths of the dominant $a_{1}(1260)$, $a_{2}(1320)$ and $\pi_{2}(1670)$ resonances are nicely reproduced by the data.

The intensity and the phase motion of the exotic wave are shown in Fig. 4. Its phase relative to the $2^{-+} \pi_{2}(1670)$ wave reproduces the phase of the $\pi_{2}$, which indicates that the exotic wave is non-resonant. The analysis shows that less than $2 \%$ of the total intensity is due to the $1^{-+}$exotic wave, so CLAS uses these preliminary data to set limits on the production of the $\pi_{1}(1600)$ in photoproduction.

\section{Outlook}

The spectrum of mesons below $c \bar{c}$ threshold is being actively investigated in both collider and fixed target experiments. BES III and COMPASS both have already accumulated huge statistical samples and will continue to take data to probe the meson spectrum with unprecedented detail. Jefferson Lab is completing its upgrade of the accelerator to $12 \mathrm{GeV}$, along with new installations to study meson spectroscopy. GlueX [23] in Hall D, which is expected to start commissioning in fall of 2014, and the Forward Tagger Facility [24] for CLAS12 will add the capability to study the meson spectrum using linearly polarized real and virtual photons. The global goal with all these facilities is to reveal the internal structure of excited mesons to shed light on the nature of gluonic interactions.

I would like to especially thank Matt Sheppherd (BESIII collaboration ), Frank Nerling (COMPASS collaboration) and Paul Eugenio (CLAS collaboration) for guidance and access to material presented in this talk. This work was supported by DOE Contract No. DE-AC05-06OR23177, under which Jefferson Science Associates, LLC, operates Jefferson Laboratory. 


\section{References}

[1] E. Klempt, A. Zaitsev, Phys. Rept. 454, 1 (2007), 0708.4016

[2] R.L. Jaffe, Phys. Rev. D 15, 267 (1977)

[3] V. Crede, C. Meyer, Prog. Part. Nucl. Phys. 63, 74 (2009), 0812.0600

[4] C. Meyer, Y. Van Haarlem, Phys. Rev. C 82, 025208 (2010), 1004.5516

[5] M. Ablikim et al. (BESIII Collaboration), Nucl. Instrum. Meth. A 614, 345 (2010), 0911.4960

[6] D. Wei (BESIII Collaboration), Review of Light Hadron Spectra at BESIII, Moriond QCD and High Energy Interactions, March 19-16, 2013

[7] M. Ablikim et al. (BESIII Collaboration), Chinese Phys. Rev. C 34, 421 (2010), 1001.5328

[8] J. Bai et al. (BES Collaboration), Phys. Rev. Lett. 91, 022001 (2003), hep-ex/0303006

[9] M. Ablikim et al. (BESIII Collaboration), Phys. Rev. Lett. 108, 112003 (2012), 1112.0942

[10] M. Ablikim et al. (BESIII Collaboration), Phys. Rev. Lett. 106, 072002 (2011), 1012. 3510

[11] Y. Chen, A. Alexandru, S. Dong, T. Draper, I. Horvath et al., Phys. Rev. D 73, 014516 (2006), hep-lat/0510074

[12] J.J. Dudek, R.G. Edwards, P. Guo, C.E. Thomas (2013), 1309. 2608

[13] M. Ablikim et al. (BESIII Collaboration), Phys. Rev. Lett. 107, 182001 (2011), 1107.1806

[14] M. Alekseev et al. (COMPASS Collaboration), Phys. Rev. Lett. 104, 241803 (2010), 0910.5842

[15] C.W. Salgado, D.P. Weygand (2013), 1310.7498

[16] F. Nerling (COMPASS Collaboration), EPJ Web Conf. 37, 01016 (2012), 1208.0487

[17] B. Mecking et al. (CLAS Collaboration), Nucl. Instrum. Meth. A 503, 513 (2003)

[18] M. Nozar et al. (CLAS Collaboration), Phys. Rev. Lett. 102, 102002 (2009), 0805.4438

[19] Jlab-Experiment-04-005 (2004), Search for New Forms of Hadronic Matter in Photoproduction, https://misportal.jlab.org/mis/physics/experiments/viewProposal.cfm?paper $\mathrm{Id}=152$

[20] S. Chung, K. Danyo, R. Hackenburg, C. Olchanski, J. Suh et al., Phys. Rev. D 65, 072001 (2002)

[21] C. Bookwalter (CLAS Collaboration), The Search for Exotic Mesons in $\gamma p \rightarrow \pi^{+} \pi^{+} \pi^{-} n$ with CLAS at Jefferson Lab, XIV International Conference on Hadron Spectroscopy, 13-17 June 2011, 1108.6112

[22] C. Bookwalter, A Search for Exotic Mesons in $\gamma p \rightarrow \pi^{+} \pi^{+} \pi-n$ with CLAS at Jefferson Lab, Ph.D. Thesis, Florida State University, Summer, 2012

[23] JLAB-Experiment-06-102 (2006), Mapping the Spectrum of Light Quark Mesons and Gluonic Excitations with Linearly Polarized Photons, https://misportal.jlab.org/mis/physics/ experiments/viewProposal. cfm?paperId=681

[24] Jlab-Experiment-11-005 (2011), Meson spectroscopy with low Q2 electron scattering in CLAS12, https://misportal.jlab.org/mis/physics/experiments/viewProposal.cfm?paper $\mathrm{Id}=637$ 